\title{
Ethyl Vanillin Activates TRPA1
}

\author{
Shaw-wen Wu, Daniel K. Fowler, Forrest J. Shaffer, Jonathon E. M. Lindberg, \\ and James $H$. Peters
}

Department of Integrative Physiology and Neuroscience, Washington State University, Pullman, Washington (D.K.F., F.J.S., J.E.M.L., J.H.P.); and Department of Neuroscience, The Scripps Research Institute, Jupiter, Florida (S.-W.W.)

Received December 6, 2016; accepted May 19, 2017

\section{ABSTRACT}

The nonselective cation channel transient receptor potential ankryn subtype family 1 (TRPA1) is expressed in neurons of dorsal root ganglia and trigeminal ganglia and also in vagal afferent neurons that innervate the lungs and gastrointestinal tract. Many TRPA1 agonists are reactive electrophilic compounds that form covalent adducts with TRPA1. Allyl isothiocyanate (AITC), the common agonist used to identify TRPA1, contains an electrophilic group that covalently binds with cysteine residues of TRPA1 and confers a structural change on the channel. There is scientific motivation to identify additional compounds that can activate TRPA1 with different mechanisms of channel gating. We provide evidence that ethyl vanillin (EVA) is a TRPA1 agonist. Using fluorescent calcium imaging and whole-cell patch-clamp electrophysiology on dissociated rat vagal afferent neurons and
TRPA1-transfected COS-7 cells, we discovered that EVA activates cells also activated by AITC. Both agonists display similar current profiles and conductances. Pretreatment with A967079, a selective TRPA1 antagonist, blocks the EVA response as well as the AITC response. Furthermore, EVA does not activate vagal afferent neurons from TRPA1 knockout mice, showing selectivity for TRPA1 in this tissue. Interestingly, EVA appears to be pharmacologically different from AITC as a TRPA1 agonist. When AITC is applied before EVA, the EVA response is occluded. However, they both require intracellular oxidation to activate TRPA1. These findings suggest that EVA activates TRPA1 but via a distinct mechanism that may provide greater ease for study in native systems compared with AITC and may shed light on differential modes of TRPA1 gating by ligand types.

\section{Introduction}

Transient receptor potential (TRP) ankyrin subtype family A 1 (TRPA1), also known as ANKTM1, is a nonselective calcium-permeable cation channel found in nociceptive sensory neurons and vagal afferent neurons (Story et al., 2003; Nagata et al., 2005). TRPA1 is predominantly coexpressed with TRP potential vanilloid subtype family 1 (TRPV1), a heat-sensitive TRP channel, in small- and medium-diameter sensory neurons of dorsal root ganglia and trigeminal ganglia (Story et al., 2003; Kobayashi et al., 2005). The vagal afferent neurons that coexpress TRPA1 and TRPV1 are unmyelinated $\mathrm{C}$-type fibers or lightly myelinated $\mathrm{A} \delta$-type fibers that sense temperature, potently initiate cardiorespiratory and gastrointestinal reflexes, and noxious stimuli to convey sensations of pain (Story et al., 2003; Kobayashi et al., 2005; Fajardo et al., 2008; Nassenstein et al., 2008; Taylor-Clark et al., 2008; Brierley et al., 2009; Yu et al., 2009; Choi et al., 2011; Shen et al., 2012; Hsu et al., 2013; Lin et al., 2013; Wang et al., 2013; Lin et al., 2015; Liu et al., 2015). Receptor coexpression has been shown via messenger RNA, immunohistochemistry, and treatment with agonists and antagonists in somatosensory

This work was supported by the National Institutes of Health National Institute of Diabetes and Digestive and Kidney Diseases [Grant DK-092651].

https://doi.org/10.1124/jpet.116.239384. afferents and to a lesser degree in visceral afferents (Story et al., 2003; Kobayashi et al., 2005; Chen et al., 2008; Hondoh et al., 2010; Weller et al., 2011; Huang et al., 2012; Kamakura et al., 2013; Weng et al., 2015; Alvarez-Berdugo et al., 2016; Green et al., 2016; Su et al., 2016). Originally characterized as a detector of noxious cold temperature $\left(<17^{\circ} \mathrm{C}\right)$ (Story et al., 2003), current studies now establish that TRPA1 thermosensitivity is species specific: warm sensing in insects and cold sensing in rats and mice, but unresponsive to temperature in primates (Viswanath et al., 2003; Chen et al., 2008; Sokabe et al., 2008; Chen and Kym, 2009; Gracheva et al., 2010; Zhong et al., 2012). Furthermore, TRPA1 can be manipulated to detect warmer temperatures, potentially causing increasing sensitivity to heat and thermal hyperalgesia (Everaerts et al., 2011; Jabba et al., 2014; Moparthi et al., 2016).

TRPA1 is activated by many pungent compounds derived from plants such as allyl isothiocyanate (AITC), allicin, wasabi, and cinnamaldehyde (Bandell et al., 2004; Jordt et al., 2004; Bautista et al., 2005; Macpherson et al., 2005, 2007). Most TRPA1 agonists are characterized as reactive electrophiles, undergoing reversible covalent modification with cysteine and lysine residues on the ankyrin repeat domains of TRPA1 (Hinman et al., 2006; Macpherson et al., 2007). This characteristic can significantly affect TRPA1 channel gating by prolonging the open configuration of the TRPA1 channel, causing pain and neurogenic inflammation in

ABBREVIATIONS: AITC, allyl isothiocyanate; AM, acetoxymethyl ester; CAP, capsaicin; DMEM, Dulbecco's modified Eagle's medium; EVA, ethyl vanillin; GFP, green fluorescent protein; HEK, human embryonic kidney; KO, knockout; TRP, transient receptor potential; TRPA1, TRP ankryn subtype family 1; TRPV1, TRP vanilloid subtype family 1 . 
nociceptive neurons (Bautista et al., 2006; Hinman et al., 2006; Macpherson et al., 2007; Paulsen et al., 2015). However, not all TRPA1 agonists are reactive electrophiles, and some agonists are nonelectrophilic, activating TRPA1 via traditional orthosteric ligand/receptor binding (Riera et al., 2009; Ryckmans et al., 2011; Del Prete et al., 2015). Thus, current efforts are underway to fully understand TRPA1 gating and synthesize or identify agonists that activate via distinct mechanisms.

Selectivity of TRP channel agonists is also important when studying TRP channels in native tissues. TRPA1 shares many agonists with TRPV3 in particular (Macpherson et al., 2006; $\mathrm{Xu}$ et al., 2006), including 2-aminoethoxy diphenylborinate (2-APB), eugenol, carvachol, camphor, cinnamaldehyde, and vanillin (Bandell et al., 2004; Jordt et al., 2004; Macpherson et al., 2005, 2006, 2007; Lubbert et al., 2013). Ethyl vanillin (EVA), another TRPV3 agonist, is 5-10 times more potent compared with vanillin in terms of TRPV3 activation, but its activation profile toward TRPA1 has not been explored (Xu et al., 2006). Although EVA can pharmacologically identify TRPV3, it also stimulates a large subpopulation of vagal afferent neurons in TRPV3 knockout (KO) mice (Wu et al., 2016). This led us to hypothesize that EVA had additional activity at other, more abundant, TRP channels in vagal afferent neurons.

In this study, we provide evidence that EVA is an agonist at TRPA1. Using calcium imaging and whole-cell voltage clamp, we show complete overlap of AITC and EVA sensitivity in vagal afferent neurons and TRPA1-transfected COS-7 cells in terms of activation profiles. In addition, EVA predicts the magnitude of AITC response in vagal afferent neurons and TRPA1-transfected COS-7 cells. Pretreatment with A967079, a selective TRPA1 antagonist, also inhibits EVA. Lastly, EVA dose-dependently stimulates dissociated neurons in rat and wild-type (Wu et al., 2016), but not TRPA1 KO mice. Furthermore, there are two pharmacologically distinct populations of EVA-sensitive neurons in which increased sensitivity to EVA also predicts sensitivity to AITC. AITC application before EVA prevents the EVA response, but not vice versa, in vagal afferent neurons and TRPA1-transfected COS-7 cells, yet they both require intracellular oxidation to induce currents. Taken together, these findings demonstrate that EVA directly stimulates TRPA1 and can identify TRPA1 in native tissues with a relatively high degree of selectivity.

\section{Materials and Methods}

Animals. Male Sprague Dawley rats (120-250 g; Simonsen Laboratories, Gilroy, CA) and male C57BL/6 mice (20-30 g) and TRPA1 KO mice (20-30 g) (B6.129P-Trpa1tm1Kykw/J; Jackson Laboratories, Bar Harbor, ME) were used under procedures approved by the Institutional Animal Care and Use Committee at Washington State University and in accordance with the Guide for the Care and Use of Laboratory Animals. Animals were housed under 12-hour light/12hour dark conditions and fed standard pellet chow ad libitum. Transgenic mice were genotyped using the protocol for separated polymerase chain reaction using primer pairs suggested by Jackson Laboratories. Genomic DNA was isolated from tail fragments using $\mathrm{NaOH}$ extraction. Genotyping of DNA from mouse tail fragments confirmed the identity of transgenic null mice.

Nodose Ganglia Isolations and Primary Neuronal Cultures. For primary rat and mouse neuronal cultures, we removed and pooled both the left and right nodose using aseptic surgical protocols previously reported (Kinch et al., 2012). Using high-magnification and blunt dissection, the vagal trunk was dissociated from the common carotid artery. All surgeries and euthanasia were performed under a deep plane of anesthesia (ketamine, $25 \mathrm{mg} / 100 \mathrm{~g}$; with xylazine, $2.5 \mathrm{mg} / 100 \mathrm{~g}$ ). Once isolated, nodose ganglia was digested in $\mathrm{Ca}^{2+} / \mathrm{Mg}^{2+}$-free Hank's balanced salt solution containing $1 \mathrm{mg} / \mathrm{mL}$ both dispase II (Hoffmann-La Roche Indianapolis, IA) and collagenase type $1 \mathrm{~A}$ (Sigma-Aldrich, St. Louis, MO) $\left(90\right.$ minutes at $37^{\circ} \mathrm{C}$ in $95 \%$ air $/ 5 \% \mathrm{CO}_{2}$ ). Following enzymatic digestion, the neurons were dissociated by gentle trituration through silicanized pipettes and then washed in Dulbecco's modified Eagle's medium (DMEM) supplemented with $10 \%$ fetal bovine serum and $1 \%$ penicillin-streptomycin. Dissociated neurons were plated onto poly-lysine-coated coverslips maintained in DMEM + $10 \%$ fetal bovine serum $\left(37^{\circ} \mathrm{C}\right.$ in $95 \%$ air $\left./ 5 \% \mathrm{CO}_{2}\right)$ and used within $24-48$ hours of isolation for ratiometric fluorescent calcium measurements.

Molecular Cloning, COS-7 Cell Culture, and Transfection. To generate TRPA1-green fluorescent protein (GFP), the full-length mouse TRPA1 coding region (National Center for Biotechnology Information accession number NM_177781.4) without stop codon was polymerase chain reaction amplified from a previously generated TRPA1 expression vector using the primers below (Zhou et al., 2013) and subcloned into the KpnI and BamHI sites of pEGFP-N1 (Clontech, Mountain View, CA).

Primer sequences: 5'-GGAGGGTACCGCCACCATGAAGCGCGGCTTGAGGAG-3'; $5^{\prime}$ - GAAAGGATCCCCAAAGTCCGGGTGGCTAATAGAAC-3'.

Clonal COS-7 cells (American Type Culture Collection, Manassas, VA) were plated on glass coverslips at a density of 250,000 cells/well of a 6-well tissue culture plate. Human embryonic kidney (HEK)293 cells (American Type Culture Collection) were plated on glass coverslips at a density of 50,000 cells/well of a 12-well plate. COS-7 and HEK293 cells were maintained in DMEM (Invitrogen, Carlsbad, CA), 10\% fetal calf serum (Atlanta Biologicals, Flowery Branch, GA), $25 \mathrm{U} / \mathrm{ml}$ penicillin, and $25 \mu \mathrm{g} / \mathrm{ml}$ streptomycin (Sigma-Aldrich). Approximately 24 hours after plating, media was replaced with DMEM and $10 \%$ fetal calf serum without pen/strep and cells were transiently transfected overnight. COS-7 cells were transfected with $2 \mu \mathrm{g}$ TRPA1-GFP and 2.4 $\mu \mathrm{l}$ lipofectamine 2000 (Invitrogen) per well. HEK293 cells were transfected with $1 \mu \mathrm{g}$ TRPA1-GFP and $1.2 \mu \mathrm{l}$ lipofectamine 2000 per well. COS-7 cells were used for calcium imaging 16-24 hours after transfection. HEK293 cells were used for electrophysiological recordings 40-48 hours following transfection.

Ratiometric Fluorescent Calcium Measurements. Intracellular calcium concentrations were monitored using fluorescent $\mathrm{Ca}^{2+}$ indicator Fura-2 acetoxymethyl ester (AM; Molecular Probes, Eugene, OR) on an inverted Nikon Eclipse Ti microscope (Nikon Instruments, Melville, NY) with an Andor Zyla digital camera (Andor Technology, Belfast, Northern Ireland). Neurons plated on coverslips the day before were loaded with Fura-2 AM $(1 \mu \mathrm{M})$ for 1 hour, rinsed, and then mounted onto a closed chamber while constantly perfused with physiologic bath (in mM: $140 \mathrm{NaCl}, 5 \mathrm{KCl}, 2 \mathrm{CaCl}_{2}, 1 \mathrm{MgCl}_{2}, 6$ glucose, 10 HEPES with $\mathrm{pH}$ adjusted to 7.4 with Tris base). Cells loaded with Fura-2 AM were alternatively excited with 340 and $380 \mathrm{~nm}$ light with fluorescence monitored at $510 \mathrm{~nm}$. Data points were collected with Nikon Elements software at 6-second time intervals, and ratios of fluorescence intensity were converted to $\mathrm{Ca}^{2+} \mathrm{nM}$ concentrations using a standard curve. The calcium calibration curve was determined previously using a sequential calcium titration with Fura-2 in the bath. The resulting fluorescence ratios were fit to determine the binding parameters using protocols previously summarized (Helmchen, 2011). The calibration curve is then saved in the imaging software for conversion of fluorescence ratios to calcium concentrations. Drugs were perfused via a common manifold, and all manipulations were performed at room temperature. Neuron viability was confirmed through depolarization with a high potassium bath, in which $55 \mathrm{mM} \mathrm{KCl}$ was used with an equimolar reduction of $\mathrm{NaCl}$ to $90 \mathrm{mM}$. 
Whole-Cell Patch-Clamp Recordings. Recording electrodes (3-4.5 $\mathrm{M} \Omega$ ) were filled with $\mathrm{CsCl}$ internal containing the following $(\mathrm{mM}): 10 \mathrm{CsCl}, 130 \mathrm{Cs}-m$ ethanesulfonate, $11 \mathrm{EGTA}, 1 \mathrm{CaCl}_{2}$, $1 \mathrm{MgCl}_{2}, 10 \mathrm{HEPES}, 2 \mathrm{Na}_{2} \mathrm{ATP}$, and $0.2 \mathrm{Na}_{2} \mathrm{GTP}$. The intracellular solution was $\mathrm{pH} 7.4$ and $285-295 \mathrm{mOsm}$. HEK293 cells were visually identified as transfected with TRPA1-GFP using an Eclipse FN1 microscope with an Intensilight C-HGFI light source filtered at $488 \mathrm{~nm}$. We studied all cells under voltage-clamp conditions with a
MultiClamp 700B or AxoClamp 200A amplifier (Molecular Devices, Union City, CA) held initially at $\mathrm{V}_{\mathrm{m}}=-60 \mathrm{mV}$ using pipettes in whole-cell patch configuration. Current-voltage relationships were generated using a voltage step protocol: $\mathrm{V}_{\text {hold }}=-60 \mathrm{mV}$ with $500 \mathrm{~ms}$ steps to $-100 \mathrm{mV}$ through $+80 \mathrm{mV}$ in $10 \mathrm{mV}$ increments. Signals were filtered at $10 \mathrm{kHz}$ and sampled at $30 \mathrm{kHz}$ using p-Clamp software (version 10.3; Molecular Devices). Liquid junctional potentials were not corrected.
A

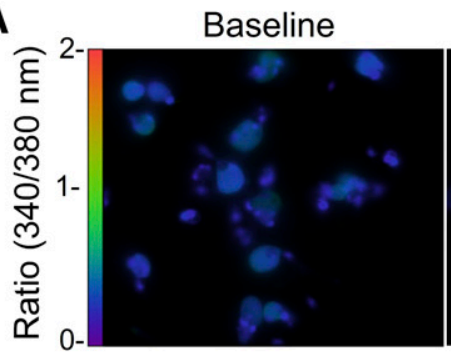

B

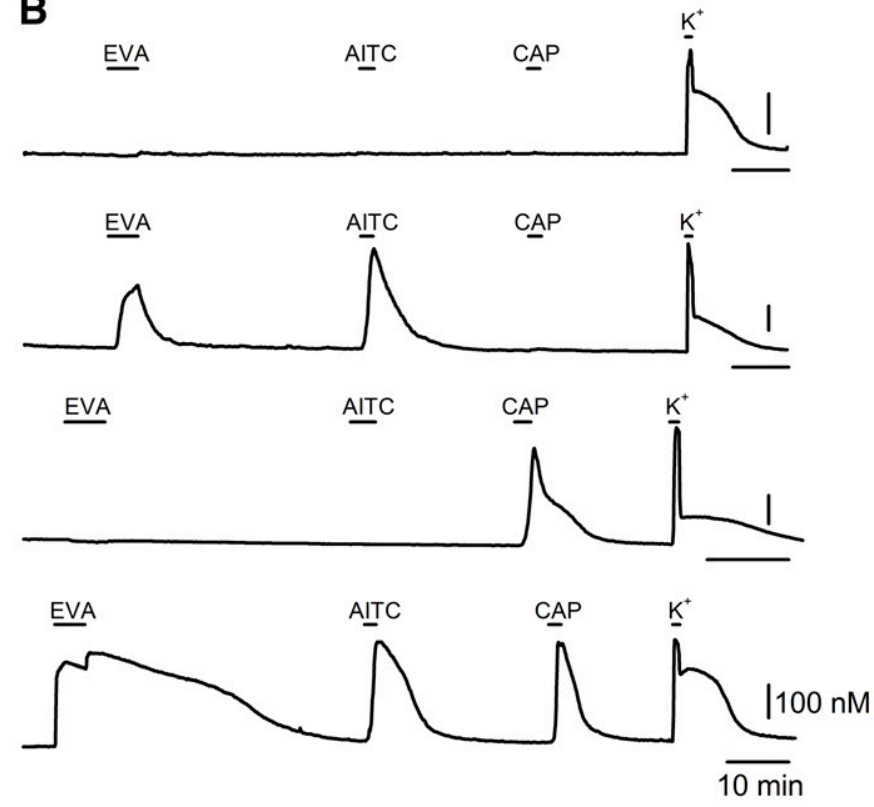

$\mathbf{F}$

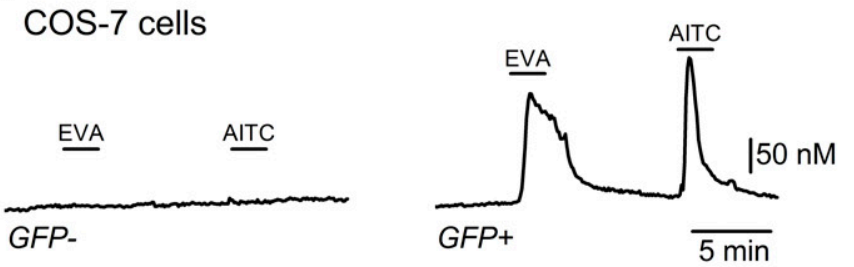

EVA (3 mM)

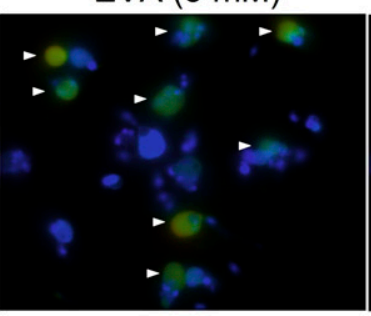

C

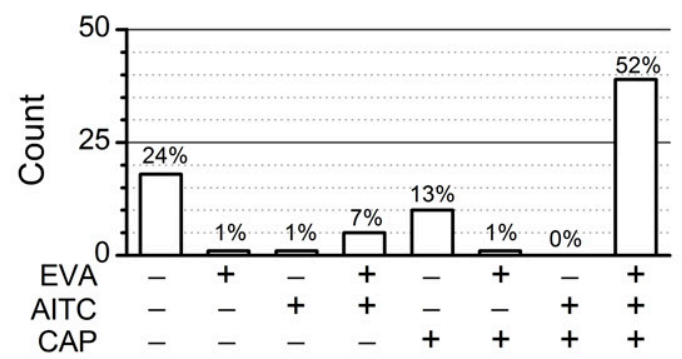

D

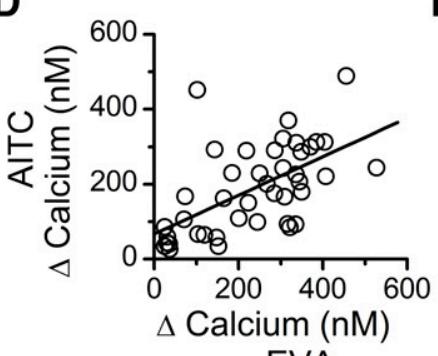

E

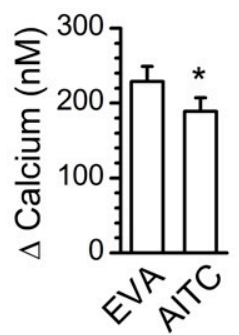

G
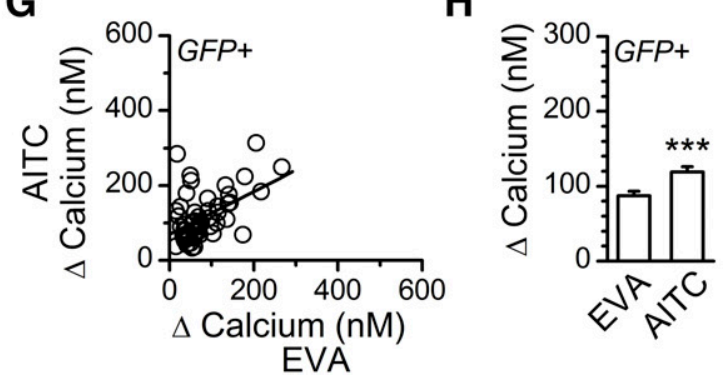

Fig. 1. EVA and AITC sensitivity overlap in rat vagal afferent neurons. EVA stimulates AITC-sensitive vagal afferent neurons and TRPA1-transfected COS-7 cells. (A) Fluorescent photomicrographs of cultured rat vagal afferent neurons loaded with Fura-2 AM calcium indicator dye at baseline and exposed to $3 \mathrm{mM}$ EVA, $300 \mu \mathrm{M}$ AITC, and $100 \mathrm{nM}$ CAP (white arrows = EVA-, AITC-, and CAP-responsive neurons; red arrows = CAP-only responsive), (B) Representative calcium traces showing response profiles of vagal afferent neurons exposed to $3 \mathrm{mM}$ EVA, $300 \mu \mathrm{M}$ AITC, and 100 nM CAP. (C) Histogram showing the percentage of neurons sensitive to EVA, AITC, and CAP ( $N=75$ neurons). (D) Scatterplot showing the positive linear relationship between EVA and AITC calcium response $\left(N=44\right.$ neurons, $\left.P<0.0001, \mathrm{R}^{2}=0.35\right)$. (E) Average change in calcium to EVA and AITC in responsive neurons. AITC responses were statistically smaller on average compared with EVA responses $(N=44, P=0.01$, paired $t$ test). (F) Calcium traces from nontransfected $\left(\mathrm{GFP}^{-}\right)$and transfected $\left(\mathrm{GFP}^{+}\right)$COS-7 cells with TRPA1-GFP. EVA- and AITC-only activated cells containing TRPA1-GFP. (G) Similar to nodose neurons, transfected COS-7 cells show a positive linear relationship between EVA and AITC calcium response $(N=83$ cells from three cultures, $P<0.0001, \mathrm{R}^{2}=0.28$ ). (H) Average change in calcium to EVA and AITC in transfected COS-7 cells. AITC responses were statistically larger on average compared with EVA responses $\left(N=83 / 3, P<0.001\right.$, Wilcoxon). ${ }^{*} P<0.05, * * * P<0.001$. 
Statistical Analysis. Data for each experiment were collected from three or more biologic replicates. When possible, we designed within-subject protocols and analyzed data using paired $t$ tests or repeated-measures analysis of variance, followed by post hoc comparisons against control. Linear correlations were generated with a linear regression fit regimen. Data are expressed as the average \pm S.E.M., with all statistical analysis performed using SigmaStat software (Systat Software, San Jose, CA).

Reagents. The following reagents were purchased from SigmaAldrich: AITC (203408-sample-K), EVA (W246409), and capsaicin (CAP; M2028). A967079 (A96), a selective TRPA1 antagonist (4716), was purchased from Tocris (Minneapolis, MN).

\section{Results}

\section{EVA and AITC Activation Overlaps in Vagal Afferents and TRPA1-Transfected COS-7 Cells}

In a recent study using fluorescent calcium imaging on dissociated TRPV3 KO mouse vagal afferent neurons, we observed that EVA still activated many neurons (Wu et al., 2016), even though TRPV3 is the only reported target of EVA (Xu et al., 2006). This suggested the presence of an additional calcium influx pathway stimulated by EVA. We used calcium imaging on primary cultures of rat vagal afferent neurons (Fig. 1A) and measured increases in intracellular calcium concentrations following exposure to $3 \mathrm{mM} \mathrm{EVA}, 300 \mu \mathrm{M}$ AITC, and $100 \mathrm{nM}$ CAP (a selective agonist for TRPV1) (Fig. 1B). We calculated the percentage of cells that responded to EVA, AITC, and CAP in vagal afferent neurons for all animal subjects (Fig. 1C). At these doses, we found near-complete overlap of EVA and AITC sensitivity, with most of these neurons also sensitive to CAP. When EVA responses were plotted against AITC response, there was a positive linear relationship (Fig. 1D). The average peak EVA response was slightly but statistically larger than the average peak AITC response (Fig. 1E). We considered the native codistribution and correlated responses as consistent with EVA and AITC targeting the same receptor and very likely TRPA1. To test this hypothesis, we transfected clonal COS-7 cells with full-length mouse TRPA1 C-terminally fused to GFP
(TRPA1-GFP) and exposed them to EVA (3 mM) and AITC $(300 \mu \mathrm{M})$. EVA and AITC only activated transfected $\left(\mathrm{GFP}^{+}\right)$ cells (Fig. 1F), showing that wild-type COS-7 cells lack endogenous TRPA1 and other potential EVA-induced calcium influx pathways such as TRPV3. $\mathrm{GFP}^{+}$cells exhibited a similar positive relationship in the size of the calcium responses between EVA and AITC (Fig. 1G). However, in the clonal cells, the peak AITC response was somewhat larger than the peak EVA response (Fig. $1 \mathrm{H}$ ).

Whole-cell voltage-clamp recordings of dissociated vagal afferent neurons confirmed EVA and AITC activity overlap in nodose neurons (Fig. 2A), with both EVA and AITC producing strong inward currents in sensitive cells (Fig. 2B). Furthermore, current-voltage relationships revealed that EVA and AITC produced approximately the same magnitude of current in sensitive cells (Fig. 2, C and D) and that these curves have similar conductance in comparison with baseline (Fig. 2E). Data were normalized to cell capacitance given the wide range of neuron size. Taken together, these data demonstrate that EVA has a similar conductance profile as AITC and activates an overlapping population of vagal afferent neurons.

EVA Activates TRPA1. To test the hypothesis that TRPA1 mediates the EVA response, we investigated the ability of the TRPA1-selective antagonist A967079 to eliminate EVA responses using calcium imaging. Antagonist pretreatment eliminated the intracellular calcium influx to EVA and in some instances lowered the basal calcium level (Fig. 3A), whereas time-matched control exposures to EVA did not show statistically significant agonist-induced desensitization of the response (first $=85 \pm 20 \mathrm{nM}$ versus second $=68 \pm$ $16 \mathrm{nM}, N=9$ neurons, $P=0.28$, paired $t$ test). Order of drug exposure was reversed for AITC because multiple exposures diminished the AITC-induced activation (first $=130 \pm 10 \mathrm{nM}$ versus second $=10 \pm 2 \mathrm{nM}, N=23$ neurons, $P<0.001$, paired $t$ test). Pretreatment with A967079 eliminated the response to AITC (Fig. 3A), consistent with both agonists activating TRPA1. Summary data are quantified in Fig. 3B. These experiments were replicated in COS-7 cells transfected with TRPA1-GFP (graphical data not shown). Confirming the findings in the native neurons, pretreatment with A967079
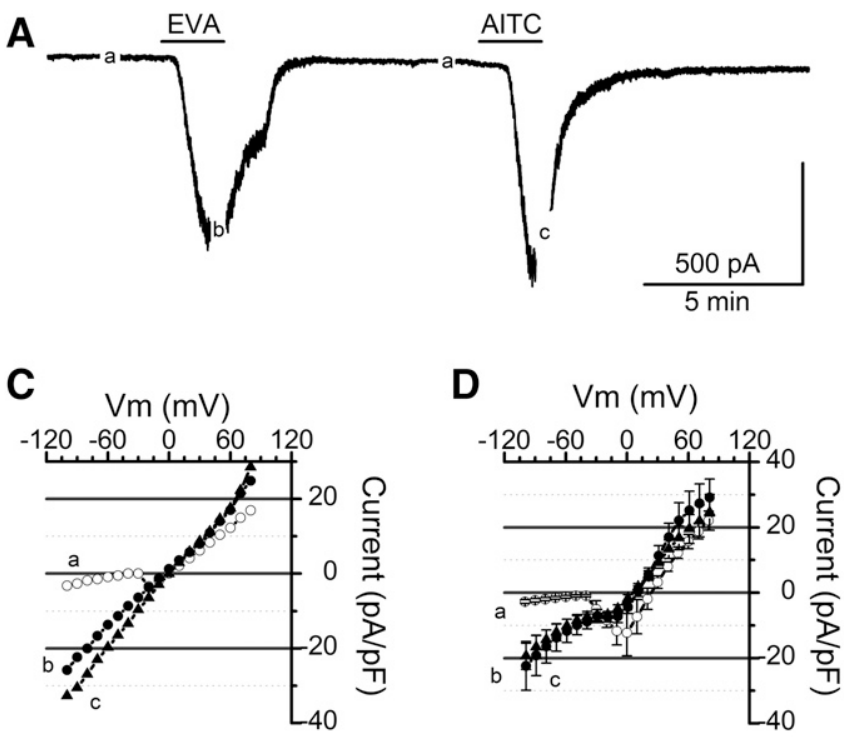

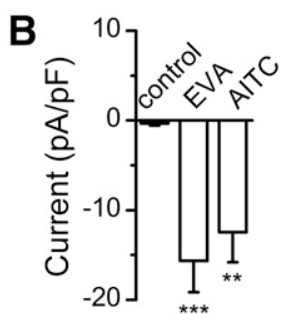

E

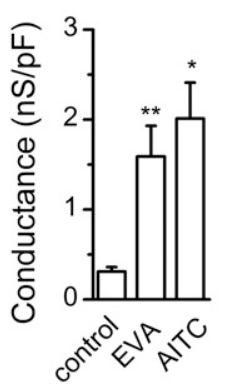

Fig. 2. EVA and AITC produce similar currentvoltage profiles in rat vagal afferent neurons. (A) Representative current trace of an individual neuron at baseline (a), responsive to $3 \mathrm{mM}$ EVA (b) and $300 \mu \mathrm{M}$ AITC (c). (B) Average current densities normalized to capacitance of $3 \mathrm{mM}$ EVA and $300 \mu \mathrm{M}$ AITC held at $-60 \mathrm{mV}(N=11$ neurons, $P=0.001$ and $P=0.005$, respectively; paired $t$ test against baseline). (C) Representative currentvoltage (I-V) relationship curve of one cell at baseline (a) and in response to EVA (b) and AITC (c) across voltages. (D) Average I-V relationship curve of neurons that respond to EVA (b) and AITC (c) compared with baseline (a), showing increased inward current due to EVA and AITC exposure ( $N=11$ neurons). (E) Average conductance normalized to capacitance of all cells responsive to EVA and AITC ( $N=11$ neurons, $P=0.003$ and $P=0.018$, respectively, paired $t$ test against baseline). Data are expressed as average \pm S.E.M. $* * P<0.01, * * * P<0.001$. 

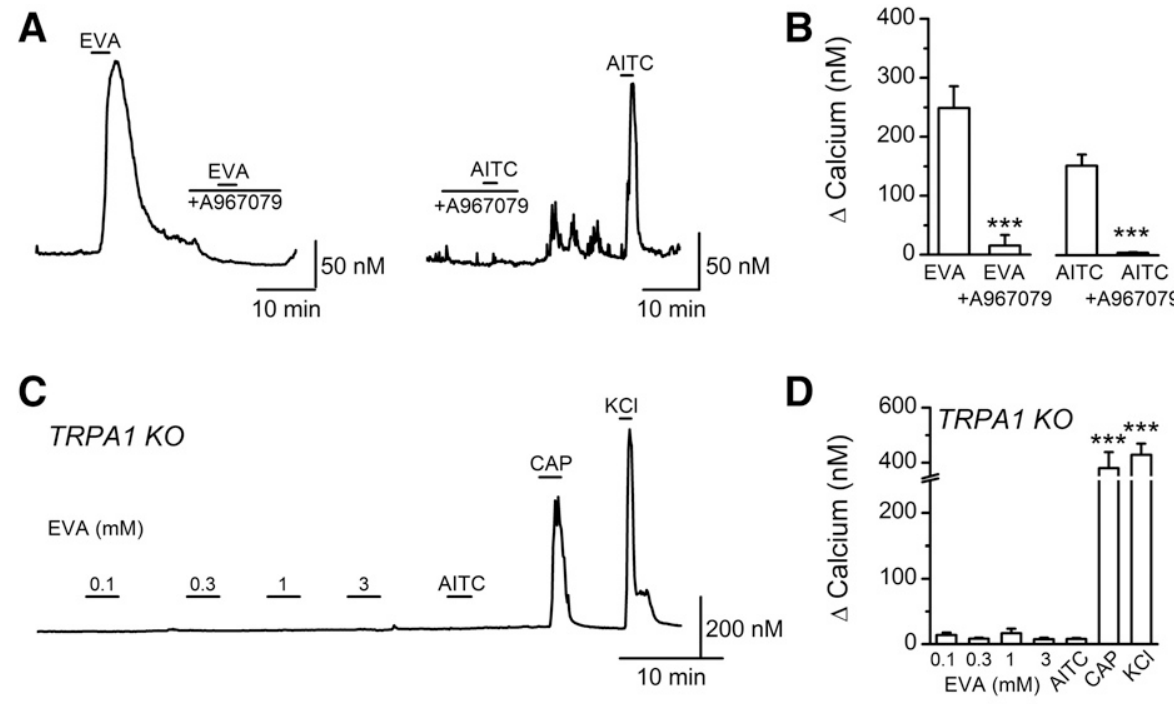

$\mathbf{E}$

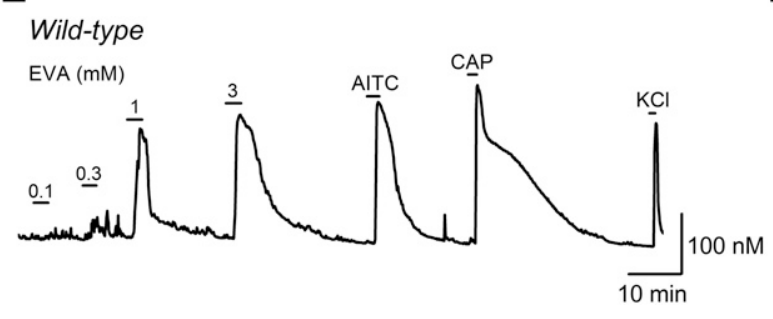

$\mathbf{F}$

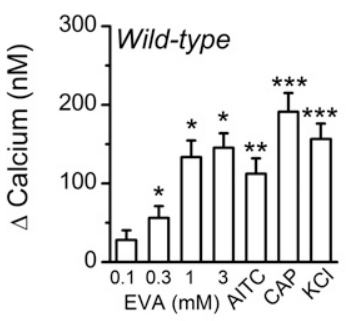

Fig. 3. Pharmacological and genetic evidence that EVA is a TRPA1 agonist. Pretreatment with the selective TRPA1 antagonist A967079 eliminates the AITC and EVA responses. (A) Representative calcium traces comparing EVA ( $3 \mathrm{mM}$ ) and AITC $(300 \mu \mathrm{M})$ treatment responses before and after pretreatment with $1 \mu \mathrm{M}$ A967079. (B) Average EVA and AITC increase in cytosolic calcium before and after pretreatment with A967079 (EVA: $N=31$ neurons, $P<0.001$, paired $t$ test; AITC: $N=12$ neurons, $P<0.001$, paired $t$ test). (C and D) EVA did not stimulate any vagal afferent neurons from TRPA1 KO mice. (C) Representative calcium traces from TRPA1 KO mice showing lack of responses to increasing concentrations of EVA $(100 \mu \mathrm{M}$ to $3 \mathrm{mM}$ ) and high-dose AITC $(300 \mu \mathrm{M})$. (D) Average change in intracellular calcium levels across EVA dose from all neurons $(N=25$ neurons). EVA and AITC failed to produce statistically significant changes in cytosolic calcium (EVA: $P=0.10$, analysis of variance and AITC: $P=0.34, t$ test), whereas CAP and potassium depolarization did (CAP: $P<0.001, t$ test and KCl: $P<0.001, t$ test). (E) EVA activated vagal afferent neurons taken from wild-type mice that were also activated by AITC. (F) Average change in intracellular calcium levels across EVA dose response for activated neurons ( $N=23$ neurons). EVA and AITC produced statistically significant changes in cytosolic calcium (EVA: $P<0.001$, analysis of variance and AITC: $P=0.002, t$ test), along with CAP and potassium depolarization (CAP: $P<0.001, t$ test and $\mathrm{KCl}: P<0.001, t$ test). Black lines indicate time of ligand application. $* P<0.05, * * P<0.01, * * * P<0.001$. completely prevented AITC- and EVA-induced calcium influx in $\mathrm{GFP}^{+}$COS-7 cells (AITC control $=105 / 123$ cells, 85\% response rate versus $\mathrm{A} 967079=0 / 78,0 \%$ response rate; $\mathrm{EVA}$ control $=103 / 176,59 \%$ response rate versus A967079 $=0 / 83$, $0 \%$ response rate). Crucially, neither EVA nor AITC activated cultured vagal afferent neurons taken from mice genetically lacking TRPA1 (TRPA1 KO), even though the neurons were shown to be viable through stimulation by CAP $(100 \mathrm{nM})$ and depolarization with elevated potassium (Hi-K) (Fig. 3, C and D). EVA and AITC did activate a subpopulation of vagal afferent neurons taken from wild-type mice (Fig. 3, E and F). Collectively, these results suggest EVA is a direct agonist of TRPA1.
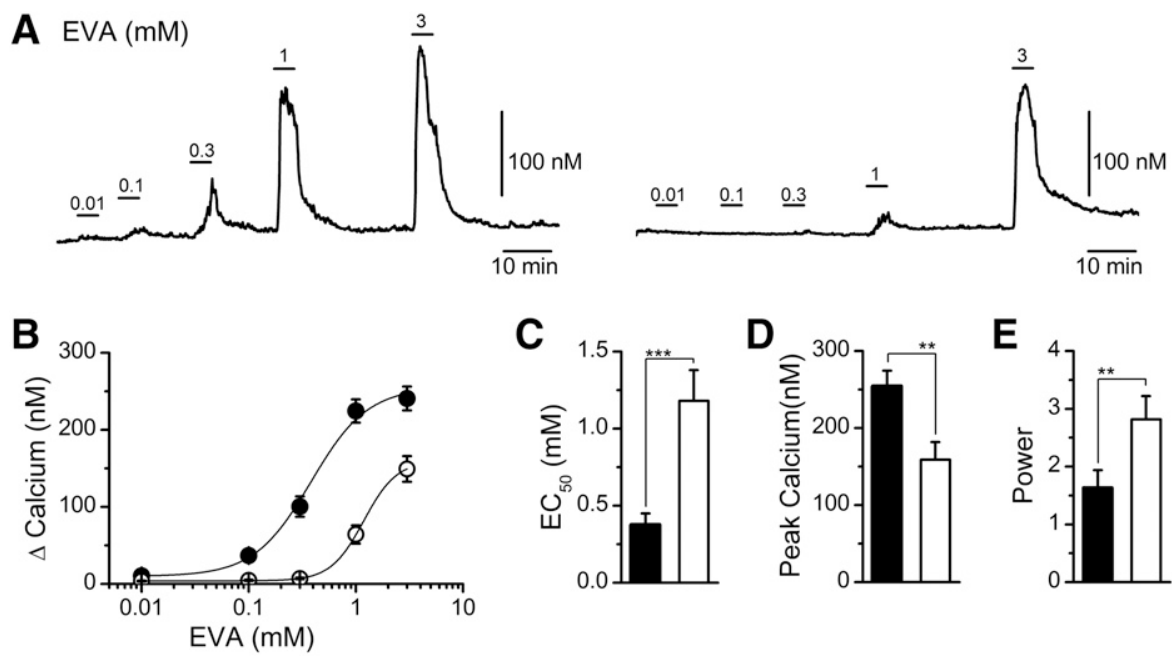

Fig. 4. Two pharmacologically distinct populations of EVA reactivity in vagal afferent neurons. Distinct profiles from EVA concentration-response curves suggest two functionally distinct groups of EVA-responsive neurons. (A) Representative calcium traces showing profiles of high-sensitivity EVAresponsive neuron (left) and a low-sensitivity EVA-responsive neuron (right). Neurons were grouped into the high-sensitivity group if they responded to $300 \mu \mathrm{M}$ EVA or less. (B) EVA dose-response curves between two different populations of EVA-sensitive neurons (dark circles: highly sensitive neurons; open circles: less sensitive neurons) (high sensitivity, $N=32$ neurons; low sensitivity, $N=31$ neurons). Group data were fit with a sigmoidal curve, and the following parameters were determined. (C) Comparison of the $\mathbf{E C}_{50}$ concentrations between high- and low-responsive populations of EVA-sensitive neurons $(P<0.001, t$ test). (D) The peak response of EVA-induced increase in cytosolic calcium $(P=0.002, t$ test). (E) The slope of the linear phase for the sigmoidal fits between groups of responsive neurons $\left(P=0.02, t\right.$ test). ${ }^{*} P<0.01,{ }^{*} * P<0.001$. 
Pharmacologically Distinct Populations of EVAResponsive Vagal Afferent Neurons. We exposed vagal afferent neurons to increasing concentrations of EVA to determine the activation kinetics for this agonist. While conducting this experiment, we noticed that EVA-responsive neurons could be grouped empirically based on the threshold concentration of EVA to which they responded (Fig. 4). Neurons that responded to EVA at $300 \mu \mathrm{M}$ or less were considered high-sensitivity neurons (Fig. 4A, left trace), whereas those that required greater than $300 \mu \mathrm{M}$ to respond were considered low-sensitivity neurons (Fig. 4A, right trace). These two populations of EVA-sensitive neurons had distinguishable concentration-response parameters when the average response data were fit with a sigmoid function (Fig. 4B). Parameters derived from these fits reveal statistically lower $\mathrm{EC}_{50}$ concentrations (Fig. 4C), higher average peak responses (Fig. 4D), and a steeper slope, measured as power, in the linear range of the fit (Fig. 4E) for the highly sensitive neurons compared with less sensitive neurons.

Extending these findings, we tested concentrationdependent activation with AITC with regard to high or low EVA sensitivity (Fig. 5). Consistent with the EVA data, we found two populations of AITC-sensitive neurons as categorized based on their sensitivity to EVA at $300 \mu \mathrm{M}$. Neurons that respond to EVA at $300 \mu \mathrm{M}$ are also more sensitive to AITC in terms of concentration-response curves (Fig. 5A, left trace), whereas neurons nonresponsive to EVA also had smaller AITC response profiles (Fig. 5A, right trace). EVA responsiveness was predictive of lower $\mathrm{EC}_{50}$ and peak responses for AITC (Fig. 5, C and D). However, the power between the two different populations of AITC responders was not statistically different (Fig. 5E). This is distinct from EVA, in which the power of neurons highly sensitive to EVA is statistically lower compared with neurons that are less sensitive to EVA. These observations differentiate two distinct populations of TRPA1-containing vagal afferent neurons.
AITC Masks the EVA Response. We observed that the order in which we bath applied EVA and AITC to vagal afferent neurons and TRPA1-GFP-transfected COS-7 cells determined EVA sensitivity and the fidelity of EVA and AITC agonist overlap. Application of high-dose EVA before application of high-dose AITC (Fig. 6A, left) yields high intracellular calcium responses to both agonists (Fig. 6B, left) and suggests complete overlap of EVA and AITC sensitivity in rat vagal afferent neurons. However, application of AITC before EVA (Fig. 6A, right) appears to prevent the subsequent EVA response, yielding only high AITC intracellular calcium response and either no EVA responses or very low EVA responses (Fig. 6B, right). The order in which we bath applied AITC and CAP to vagal afferent neurons (Fig. 6C) did not have any effect on magnitude of AITC or CAP response (Fig. 6D), suggesting the effect is specific to TRPA1. To test this phenomenon, we replicated the AITC inhibition of EVA using TRPA1-GFP-transfected COS-7 cells (Fig. 6, E and F). These data suggest EVA and AITC are both TRPA1 agonists that activate the receptor via different mechanisms such that AITC activation of TRPA1 diminishes binding or activation by EVA.

\section{AITC- and EVA-Induced Currents Are Diminished by Intracellular Oxidative Buffering}

One possible difference in the mechanism of activation between AITC and EVA was the extent to which they rely on intracellular oxidation to activate the TRPA1 channel. To investigate this possibility, we recorded AITC- and EVAinduced whole-cell currents from TRPA1-transfected HEK293 cells with control intracellular solutions or in the presence of oxidative buffer glutathione $(10 \mathrm{mM})$ (Fig. 7). We found that intracellular glutathione significantly reduced the mean AITC current compared with control recordings, consistent with diminished electrophilic interactions (Fig. 7, A and B). The EVA-induced currents were reduced to a similar extent as the
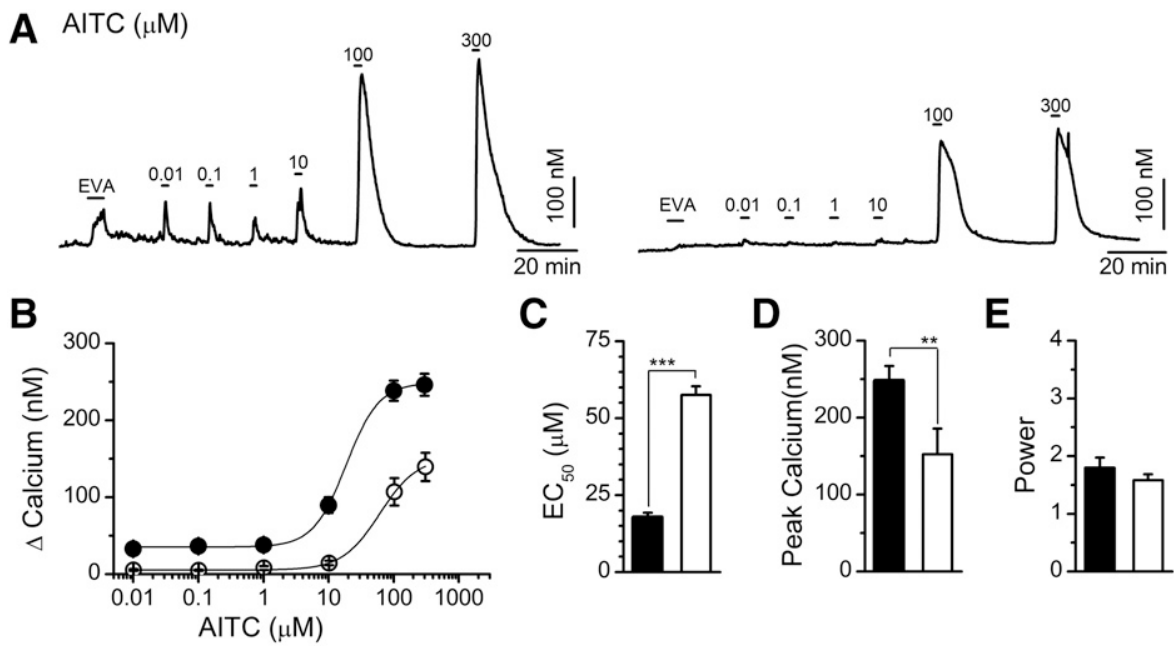

C

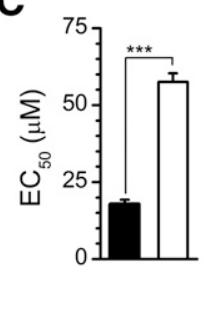

D

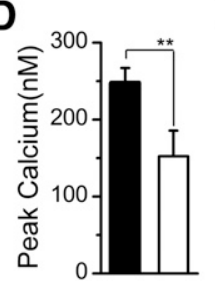

E

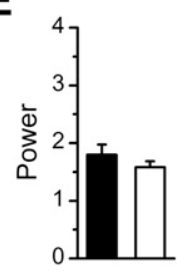

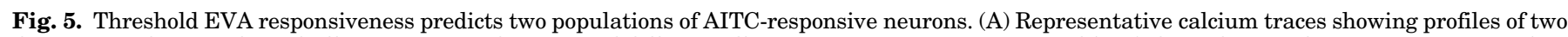

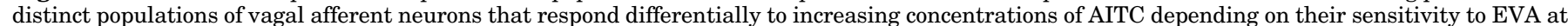

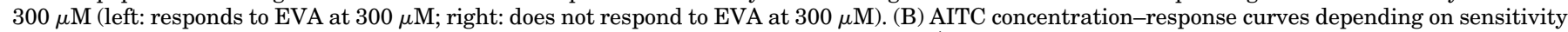

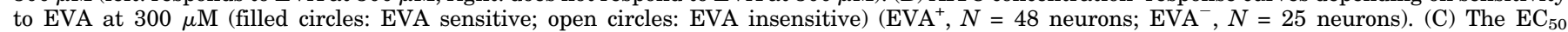

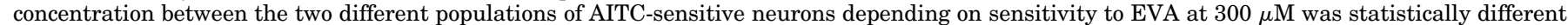

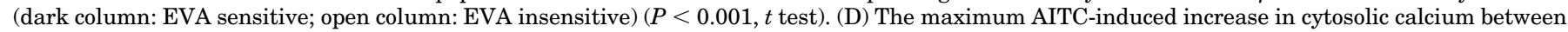

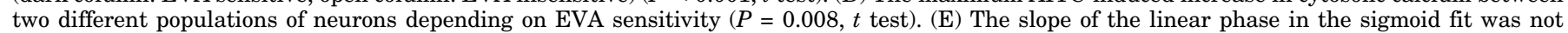
statistically different between the two AITC-responsive populations $(P=0.39, t$ test). $* * P<0.01, * * * P<0.001$. 
A

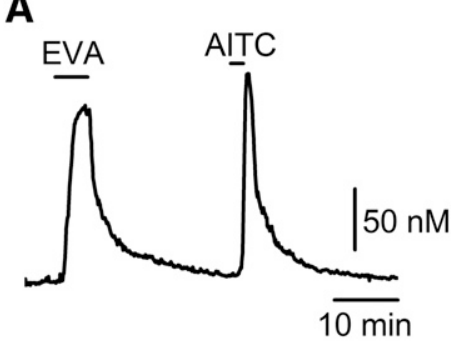

C

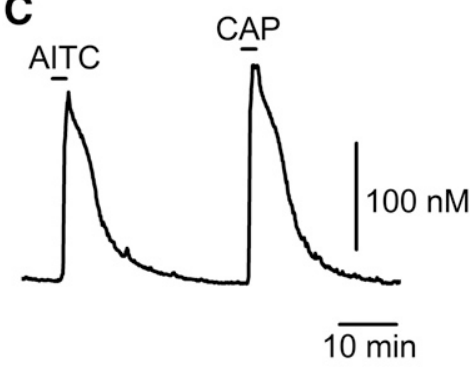

$\mathbf{E}$

COS-7 cells

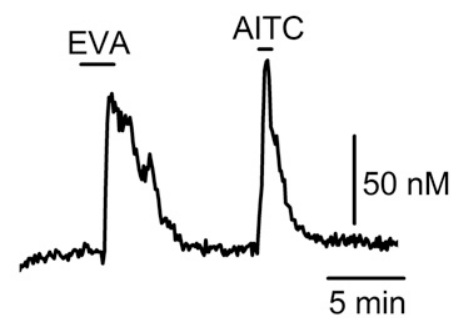

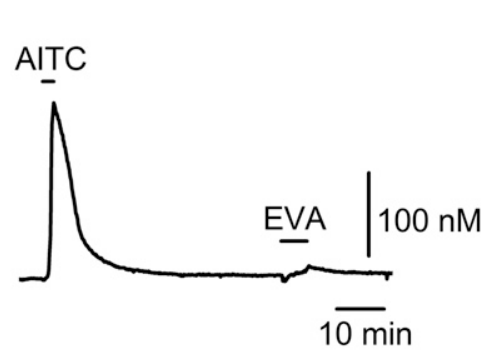

B
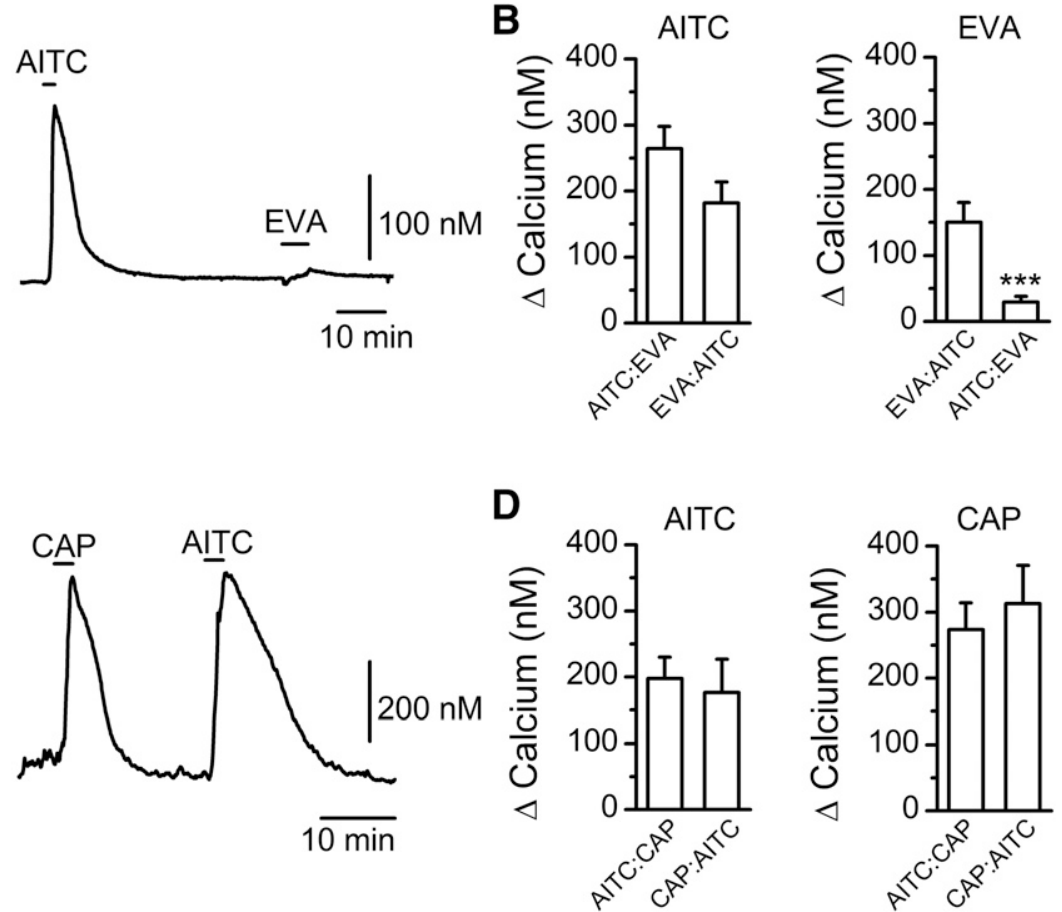

$\mathbf{F}$
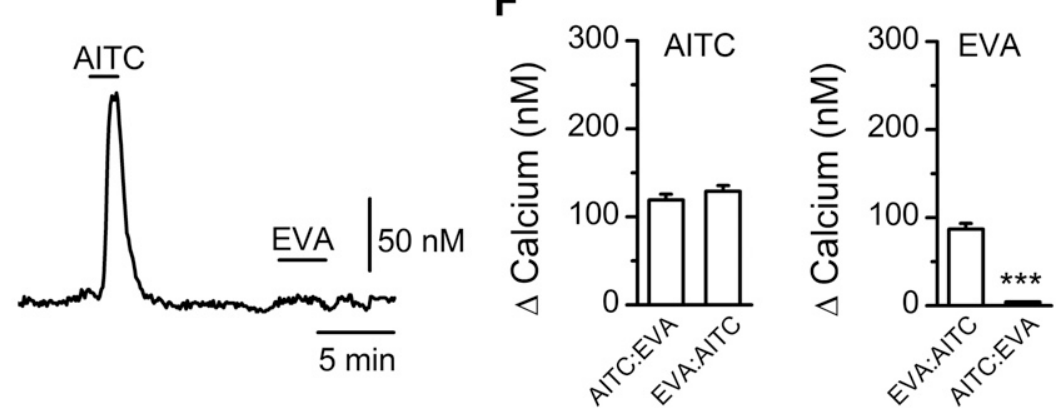

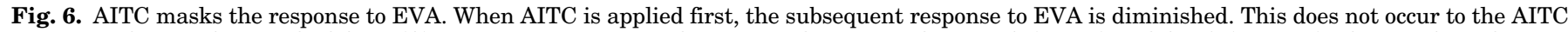

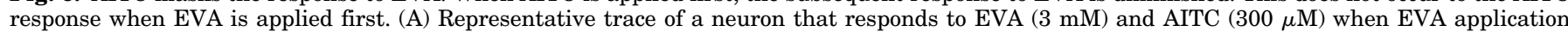

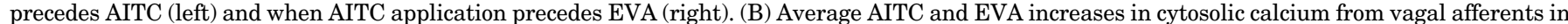

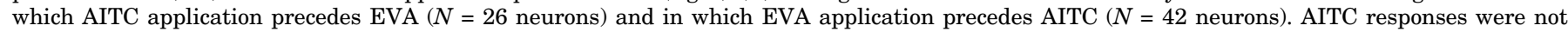

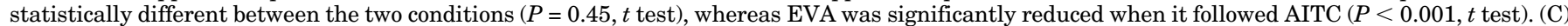

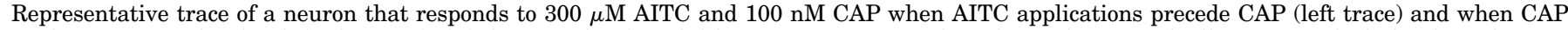

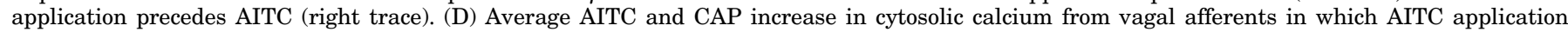

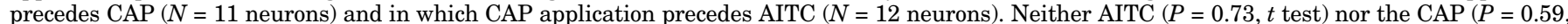

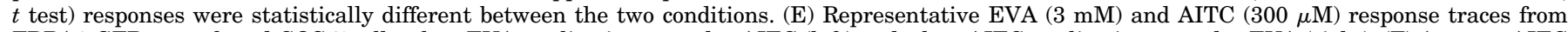

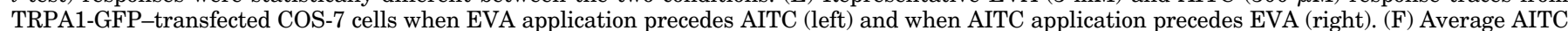

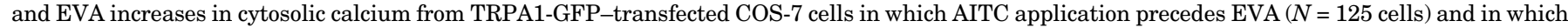

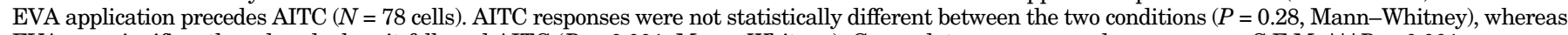
EVA was significantly reduced when it followed AITC $(P<0.001$, Mann-Whitney). Group data are expressed as average \pm S.E.M. $* * * P<0.001$.

AITC currents (Fig. 7, C and D), suggesting EVA also requires the ability to oxidize TRPA1 to activate the channel. These data suggest that differences in oxidative capacity alone do not explain differences in channel activation between AITC and EVA and might indicate additional irreversible ligand/channel interactions for AITC, which EVA lacks.

\section{Discussion}

The primary finding of this study is that EVA is an agonist of TRPA1 in native vagal afferent neurons and TRPA1GFP-transfected COS-7 cells with a distinct pharmacological profile from AITC. Using AITC as a reference agonist for
TRPA1, we show complete overlap of AITC and EVA sensitivity in vagal afferent neurons using calcium imaging and whole-cell voltage-clamp experiments. Furthermore, EVA response predicts the AITC response in vagal afferent neurons and TRPA1-GFP-transfected COS-7 cells, whereas pretreatment with A967079, a specific TRPA1 antagonist, eliminates EVA responses. EVA dose-dependently stimulates dissociated neurons in rat and wild-type mice (Wu et al., 2016), but not TRPA1 KO mice. In addition, application of increasing concentrations of EVA revealed two distinct populations of EVA-sensitive neurons with distinct kinetic parameters. Threshold of EVA sensitivity also predicted two distinct populations of AITC-sensitive neurons with characteristic 

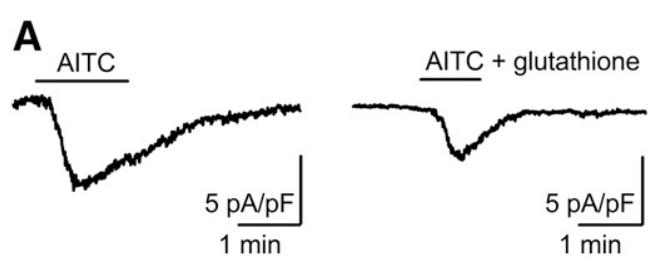

(1)
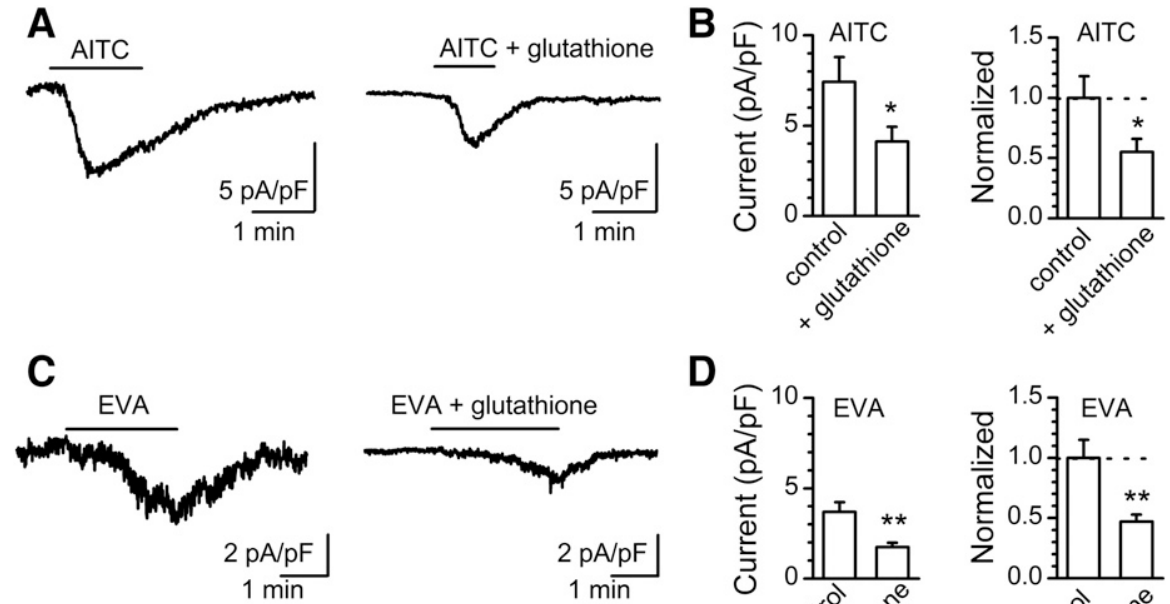

D

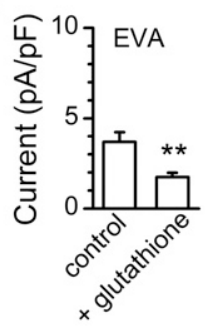

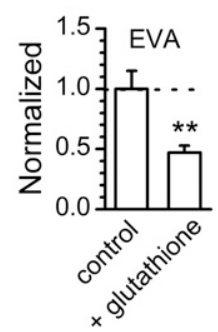

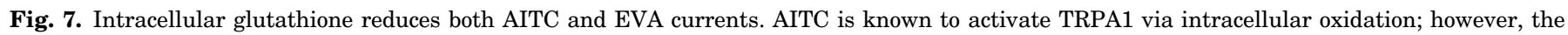

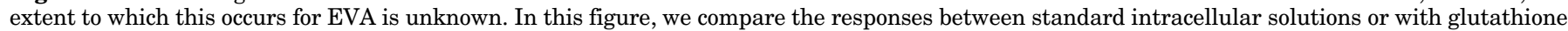

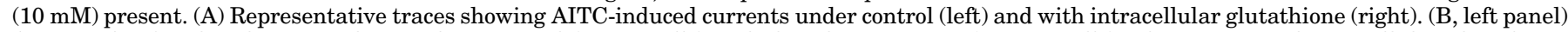

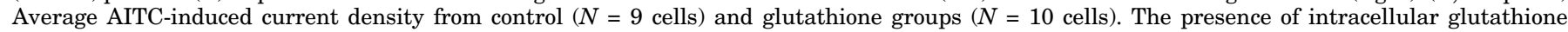

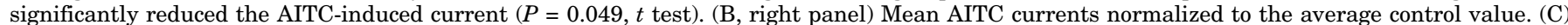

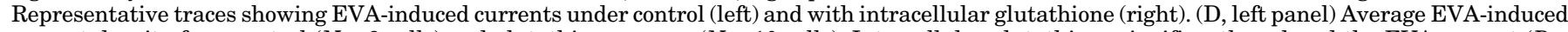

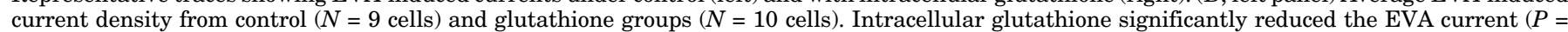

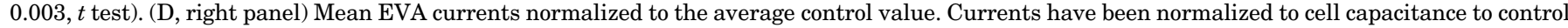
for differences in cell size. $* P<0.05$, $* * P<0.01$.

kinetic parameters. Lastly, application of AITC before EVA occludes the EVA response at a maximum concentration of $3 \mathrm{mM}$, suggesting that AITC activation alters TRPA1 in such a way to prevent binding or activation by EVA. Therefore, EVA application may be useful for investigating how TRPA1 gating is differentially affected by ligand types. EVA should also be advantageous for studying TRPA1 in vagal afferent neurons and other native tissues because EVA appears to have minimal interference with TRPA1 gating; for instance, covalent modification of TRPA1 by AITC has been associated with prolonged channel activation, causing tissue damage (Hinman et al., 2006; Macpherson et al., 2007).

TRP Channel Signaling in Vagal Afferent Neurons. There are a multitude of TRP channels that are expressed in vagal afferent neurons with a wide range of neurophysiological functions both in the periphery and at the central terminals (Wu et al., 2014). However, our understanding of the actions and interactions of many of these TRP channels in the context of vagal afferent signaling is still developing. To date, we know that TRPV1 expression at the terminals of vagal afferent neurons contributes to asynchronous and spontaneous release of glutamate, a characteristic that enables adaptive synaptic transmission (Peters et al., 2010; Shoudai et al., 2010). TRPA1 is as abundant as TRPV1 in vagal afferent neurons and seems to coexpress in TRPV1expressing neurons (Kobayashi et al., 2005; Nassenstein et al., 2008; Lin et al., 2015), yet the role of TRPA1 in afferent neurotransmitter release is currently unexplored. To study the function of TRPA1 in vagal afferent signaling, we have found a way to selectively stimulate it pharmacologically in native vagal afferent neurons via EVA, which activates TRPA1 differently than the standard TRPA1 agonist AITC.

We had originally overlooked the importance of TRPA1 in vagal afferent neurons, turning our attention toward TRP channels stimulated by warm temperatures such as TRPV3. However, EVA also stimulated a significant number of vagal afferent neurons in TRPV3 KO mice, demonstrating that EVA is not a selective TRPV3 agonist (Wu et al., 2016). Using a more selective TRPV3 agonist (farnesyl pyrophosphate), we pharmacologically determined that TRPV3 was present in vagal afferent neurons in extremely small amounts (Wu et al., 2016), and therefore was likely negligible to the overall EVA response observed in wild-type animals. These observations combined with our current findings suggest that EVA primarily targets TRPA1 in vagal afferent neurons. Crucially, whereas EVA concentration-dependently stimulated a sizable portion of vagal afferent neurons in TRPV3 KO mice, EVA did not stimulate any vagal afferent neurons in TRPA1 KO mice. This shows that EVA, at the concentrations used in this study, is not an agonist for any other calcium-conducting channel expressed in vagal afferent neurons. Although this does not preclude EVA activity at other TRP channels, the reported expression of many TRP channels in vagal afferent neurons and lack of EVA signaling suggests a fairly high degree of specificity to TRPA1 (Buniel et al., 2004; Zhang et al., 2004; Glazebrook et al., 2005; Elg et al., 2007; Staaf et al., 2010; Zhao et al., 2010). In TRPA1-GFP-transfected COS-7 cells, we found similar overlap of EVA and AITC activation, effectiveness of TRPA1 antagonism, and linearity of fit between EVA and AITC as in native vagal afferent neurons (Fig. 1). These findings strengthen the argument that EVA is an agonist of TRPA1. Our findings suggest that EVA is a selective agonist of TRPA1 in vagal afferent neurons.

Potential Importance of EVA as a TRPA1 Agonist. Many reactive compounds, similar to AITC, activate TRPA1 via covalent modification of the channel versus traditional lock and key binding. However, there are also numerous nonreactive compounds, such as menthol, that activate 
TRPA1 at alternative structural sites without covalently modulating the channel (Xiao et al., 2008). Despite these findings, the underlying mechanism of noncovalent TRPA1 agonism is relatively unknown. Several lines of evidence suggest that EVA is a TRPA1 agonist that activates TRPA1 differently than AITC. The chemical structure of EVA and AITC reveals that both compounds contain electrophilic nucleophile groups, and intracellular buffering with glutathione (Fig. 7) diminishes activation of TRPA1 by both ligands, indicating oxidation is required for increased TRPA1 gating (Materazzi et al., 2012). Yet, when AITC is applied prior to EVA, it decreases the response to EVA; but EVA does not decrease the response to AITC when applied first (Fig. 6). These observations suggest that EVA's mode of TRPA1 activation is in some way different than that of AITC and may suggest that AITC is a chemically more reactive nucleophile than EVA. There are several potential advantages to the differential gating kinetics of EVA activation. A less reactive nucleophile that presumably does not covalently bind to the receptor is less likely to irreversibly change channel confirmation and may provide advantages from an experimental standpoint as well as perhaps in the design of compounds for potential clinical use. Our experiments involving AITC required extended wash times to return the experimental system back to its original state. Perhaps via covalent modification of residues on the intracellular ankyrin binding domains or differential unbinding from orthosteric versus allosteric sites (Lane et al., 2013), AITC induces a change on the experimental system by keeping the TRPA1 channel in a ligand-bound/open or ligand-bound/inactive configuration for longer periods of time compared with EVA. As a result, EVA can be used repeatedly and requires shorter wash times. It was originally reported that vanillin, the precursor to EVA, is also a TRPA1 agonist and to a small extent TRPV1 agonist (Lubbert et al., 2013). In contrast to vanillin, EVA does not appear to be a TRPV1 agonist at the concentrations we tested because it had no effect in neurons from TRPA1 KO mice, which maintained their expression of TRPV1 (Fig. 3). Furthermore, the differences in pharmacology between AITC and vanillin have largely not been explored. Concentration-response curves of EVA and AITC reveal that there are two distinct populations of sensitive cells with distinct magnitudes of $\mathrm{EC}_{50}$, peak response, and power. These different populations may be a consequence of differential expression of TRPA1b, a TRPA1 splice variant. However, TRPA1b is not reported to be expressed in the rat nor alter the $\mathrm{EC}_{50}$ of AITC activation (Zhou et al., 2013). The difference may also arise as a product of TRPA1 expression level. More specifically, one would predict that large amounts of TRPA1 would exhibit a lower $\mathrm{EC}_{50}$ and increased apparent sensitivity to agonists compared with cells with lower amounts of TRPA1. Alternatively, additional TRP channels capable of modifying the activity of TRPA1 may be present (Hondoh et al., 2010; Everaerts et al., 2011; Weller et al., 2011; Huang et al., 2012; Lubbert et al., 2013; Wu et al., 2014; Lin et al., 2015; Weng et al., 2015). Although we identified pharmacological subpopulations of EVA- and AITC-sensitive vagal afferents, the extent to which these differences in signaling have physiologic importance is unknown.

Summary and Perspectives. The largely nonselective profile of many TRP channel agonists and antagonists makes it difficult to identify and manipulate specific TRP channels in native tissue. In this study, we were able to pharmacologically identify TRPA1 using AITC and characterize the novel agonist EVA. AITC and EVA shared similarities in terms of overlap, response rate, and conductance profiles. However, key differences in their chemical structures may explain differences in their activation of TRPA1, perhaps via differences in binding sites and/or affinity. EVA behaves as a low-affinity full agonist compared with AITC and may provide certain advantages for experimental interrogation of TRPA1.

\section{Acknowledgments}

We acknowledge BreeAnne Peterson for assistance in completing this manuscript.

\section{Authorship Contributions}

Participated in research design: Wu, Fowler, Peters.

Conducted experiments: Wu, Fowler, Shaffer, Lindberg, Peters.

Performed data analysis: Wu, Fowler, Shaffer, Lindberg, Peters.

Wrote or contributed to the writing of the manuscript: $\mathrm{Wu}$, Fowler, Peters.

\section{References}

Alvarez-Berdugo D, Rofes L, Farré R, Casamitjana JF, Enrique A, Chamizo J, Padrón A, Navarro X, and Clavé P (2016) Localization and expression of TRPV1 and TRPA1 in the human oropharynx and larynx. Neurogastroenterol Motil 28: 91-100.

Bandell M, Story GM, Hwang SW, Viswanath V, Eid SR, Petrus MJ, Earley TJ, and Patapoutian A (2004) Noxious cold ion channel TRPA1 is activated by pungent compounds and bradykinin. Neuron 41:849-857.

Bautista DM, Jordt SE, Nikai T, Tsuruda PR, Read AJ, Poblete J, Yamoah EN, Basbaum AI, and Julius D (2006) TRPA1 mediates the inflammatory actions of environmental irritants and proalgesic agents. Cell 124:1269-1282.

Bautista DM, Movahed P, Hinman A, Axelsson HE, Sterner O, Högestätt ED, Julius D, Jordt SE, and Zygmunt PM (2005) Pungent products from garlic activate the sensory ion channel TRPA1. Proc Natl Acad Sci USA 102:12248-12252.

Brierley SM, Hughes PA, Page AJ, Kwan KY, Martin CM, O'Donnell TA, Cooper NJ, Harrington AM, Adam B, Liebregts T, et al. (2009) The ion channel TRPA1 is required for normal mechanosensation and is modulated by algesic stimuli. Gastroenterology 137:2084-2095 e2083.

Buniel M, Wisnoskey B, Glazebrook PA, Schilling WP, and Kunze DL (2004) Distribution of TRPC channels in a visceral sensory pathway. Novartis Found Symp 258:236-243; discussion 243-247, 263-266.

Chen J and Kym PR (2009) TRPA1: the species difference. J Gen Physiol 133: $623-625$.

Chen J, Zhang XF, Kort ME, Huth JR, Sun C, Miesbauer LJ, Cassar SC, Neelands T, Scott VE, Moreland RB, et al. (2008) Molecular determinants of species-specific activation or blockade of TRPA1 channels. J Neurosci 28:5063-5071.

Choi MJ, Jin Z, Park YS, Rhee YK, and Jin YH (2011) Transient receptor potential (TRP) A1 activated currents in TRPV1 and cholecystokinin-sensitive cranial visceral afferent neurons. Brain Res 1383:36-42.

Del Prete D, Caprioglio D, Appendino G, Minassi A, Schiano-Moriello A, Di Marzo V, and De Petrocellis L (2015) Discovery of non-electrophilic capsaicinoid-type TRPA1 ligands. Bioorg Med Chem Lett 25:1009-1011.

Elg S, Marmigere F, Mattsson JP, and Ernfors P (2007) Cellular subtype distribution and developmental regulation of TRPC channel members in the mouse dorsal root ganglion. J Comp Neurol 503:35-46.

Everaerts W, Gees M, Alpizar YA, Farre R, Leten C, Apetrei A, Dewachter I, van Leuven F, Vennekens R, De Ridder D, et al. (2011) The capsaicin receptor TRPV1 is a crucial mediator of the noxious effects of mustard oil. Curr Biol 21:316-321.

Fajardo O, Meseguer V, Belmonte C, and Viana F (2008) TRPA1 channels mediate cold temperature sensing in mammalian vagal sensory neurons: pharmacological and genetic evidence. J Neurosci 28:7863-7875.

Glazebrook PA, Schilling WP, and Kunze DL (2005) TRPC channels as signal transducers. Pflugers Arch 451:125-130.

Gracheva EO, Ingolia NT, Kelly YM, Cordero-Morales JF, Hollopeter G, Chesler AT, Sánchez EE, Perez JC, Weissman JS, and Julius D (2010) Molecular basis of infrared detection by snakes. Nature 464:1006-1011.

Green D, Ruparel S, Gao X, Ruparel N, Patil M, Akopian A, and Hargreaves K (2016) Central activation of TRPV1 and TRPA1 by novel endogenous agonists contributes to mechanical allodynia and thermal hyperalgesia after burn injury. Mol Pain 12: $1-9$.

Helmchen F. Calibration of fluorescent calcium indicators. Cold Spring Harbor Protoc 2011: 923-930, 2011.

Hinman A, Chuang HH, Bautista DM, and Julius D (2006) TRP channel activation by reversible covalent modification. Proc Natl Acad Sci USA 103:19564-19568.

Hondoh A, Ishida Y, Ugawa S, Ueda T, Shibata Y, Yamada T, Shikano M, Murakami S, and Shimada S (2010) Distinct expression of cold receptors (TRPM8 and TRPA1) in the rat nodose-petrosal ganglion complex. Brain Res 1319:60-69.

Hsu CC, Lin RL, Lee LY, and Lin YS (2013) Hydrogen sulfide induces hypersensitivity of rat capsaicin-sensitive lung vagal neurons: role of TRPA1 receptors. Am J Physiol Regul Integr Comp Physiol 305:R769-R779. 
Huang D, Li S, Dhaka A, Story GM, and Cao YQ (2012) Expression of the transient receptor potential channels TRPV1, TRPA1 and TRPM8 in mouse trigeminal primary afferent neurons innervating the dura. Mol Pain 8:66.

Jabba S, Goyal R, Sosa-Pagán JO, Moldenhauer H, Wu J, Kalmeta B, Bandell M, Latorre R, Patapoutian A, and Grandl J (2014) Directionality of temperature activation in mouse TRPA1 ion channel can be inverted by single-point mutations in ankyrin repeat six. Neuron 82:1017-1031.

Jordt SE, Bautista DM, Chuang HH, McKemy DD, Zygmunt PM, Högestätt ED, Meng ID, and Julius D (2004) Mustard oils and cannabinoids excite sensory nerve fibres through the TRP channel ANKTM1. Nature 427:260-265.

Kamakura T, Ishida Y, Nakamura Y, Yamada T, Kitahara T, Takimoto Y, Horii A, Uno A, Imai T, Okazaki S, et al. (2013) Functional expression of TRPV1 and TRPA1 in rat vestibular ganglia. Neurosci Lett 552:92-97.

Kinch DC, Peters JH, and Simasko SM (2012) Comparative pharmacology of cholecystokinin induced activation of cultured vagal afferent neurons from rats and mice. PLoS One 7:e34755.

Kobayashi K, Fukuoka T, Obata K, Yamanaka H, Dai Y, Tokunaga A, and Noguchi K (2005) Distinct expression of TRPM8, TRPA1, and TRPV1 mRNAs in rat primary afferent neurons with adelta/c-fibers and colocalization with trk receptors. J Comp Neurol 493:596-606.

Lane JR, Sexton PM, and Christopoulos A (2013) Bridging the gap: bitopic ligands of G-protein-coupled receptors. Trends Pharmacol Sci 34:59-66.

Lin YJ, Hsu HH, Ruan T, and Kou YR (2013) Mediator mechanisms involved in TRPV1, TRPA1 and P2X receptor-mediated sensory transduction of pulmonary ROS by vagal lung C-fibers in rats. Respir Physiol Neurobiol 189:1-9.

Lin YJ, Lin RL, Ruan T, Khosravi M, and Lee LY (2015) A synergistic effect of simultaneous TRPA1 and TRPV1 activations on vagal pulmonary C-fiber afferents. J Appl Physiol (1985) 118:273-281.

Liu Z, Hu Y, Yu X, Xi J, Fan X, Tse CM, Myers AC, Pasricha PJ, Li X, and Yu S (2015) Allergen challenge sensitizes TRPA1 in vagal sensory neurons and afferent C-fiber subtypes in guinea pig esophagus. Am J Physiol Gastrointest Liver Physiol 308 G482-G488.

Lübbert M, Kyereme J, Schöbel N, Beltrán L, Wetzel CH, and Hatt H (2013) Transient receptor potential channels encode volatile chemicals sensed by rat trigeminal ganglion neurons. PLoS One 8:e77998.

Macpherson LJ, Dubin AE, Evans MJ, Marr F, Schultz PG, Cravatt BF, and Patapoutian A (2007) Noxious compounds activate TRPA1 ion channels through covalent modification of cysteines. Nature 445:541-545.

Macpherson LJ, Geierstanger BH, Viswanath V, Bandell M, Eid SR, Hwang S, and Patapoutian A (2005) The pungency of garlic: activation of TRPA1 and TRPV1 in response to allicin. Curr Biol 15:929-934.

Macpherson LJ, Hwang SW, Miyamoto T, Dubin AE, Patapoutian A, and Story GM (2006) More than cool: promiscuous relationships of menthol and other sensory compounds. Mol Cell Neurosci 32:335-343.

Materazzi S, Fusi C, Benemei S, Pedretti P, Patacchini R, Nilius B, Prenen J, Creminon C, Geppetti P, and Nassini R (2012) TRPA1 and TRPV4 mediate paclitaxel-induced peripheral neuropathy in mice via a glutathione-sensitive mechanism. Pflugers Arch 463:561-569.

Moparthi L, Kichko TI, Eberhardt M, Högestätt ED, Kjellbom P, Johanson U, Reeh PW, Leffler A, Filipovic MR, and Zygmunt PM (2016) Human TRPA1 is a heat sensor displaying intrinsic U-shaped thermosensitivity. Sci Rep 6:28763.

Nagata K, Duggan A, Kumar G, and García-Añoveros J (2005) Nociceptor and hair cell transducer properties of TRPA1, a channel for pain and hearing. J Neurosci 25: $4052-4061$

Nassenstein C, Kwong K, Taylor-Clark T, Kollarik M, Macglashan DM, Braun A and Undem BJ (2008) Expression and function of the ion channel TRPA1 in vagal afferent nerves innervating mouse lungs. $J$ Physiol 586:1595-1604

Paulsen CE, Armache JP, Gao Y, Cheng Y, and Julius D (2015) Structure of the TRPA1 ion channel suggests regulatory mechanisms. Nature 520:511-517.

Peters JH, McDougall SJ, Fawley JA, Smith SM, and Andresen MC (2010) Primary afferent activation of thermosensitive TRPV1 triggers asynchronous glutamate release at central neurons. Neuron 65:657-669.

Riera CE, Menozzi-Smarrito C, Affolter M, Michlig S, Munari C, Robert F, Vogel H, Simon SA, and le Coutre J (2009) Compounds from Sichuan and Melegueta peppers activate, covalently and non-covalently, TRPA1 and TRPV1 channels. $\mathrm{Br} J$ Pharmacol 157:1398-1409.

Ryckmans T, Aubdool AA, Bodkin JV, Cox P, Brain SD, Dupont T, Fairman E, Hashizume Y, Ishii N, Kato T, et al. (2011) Design and pharmacological evaluation of PF-4840154, a non-electrophilic reference agonist of the TrpA1 channel. Bioorg Med Chem Lett 21:4857-4859.

Shen MY, Luo YL, Yang CH, Ruan T, and Lai CJ (2012) Hypersensitivity of lung vagal $C$ fibers induced by acute intermittent hypoxia in rats: role of reactive oxygen species and TRPA1. Am J Physiol Regul Integr Comp Physiol 303:R1175-R1185.

Shoudai K, Peters JH, McDougall SJ, Fawley JA, and Andresen MC (2010) Thermally active TRPV1 tonically drives central spontaneous glutamate release. $J$ Neurosci 30:14470-14475.

Sokabe T, Tsujiuchi S, Kadowaki T, and Tominaga M (2008) Drosophila painless is a $\mathrm{Ca} 2+$-requiring channel activated by noxious heat. $J$ Neurosci 28:9929-9938.

Staaf S, Franck MC, Marmigère F, Mattsson JP, and Ernfors P (2010) Dynamic expression of the TRPM subgroup of ion channels in developing mouse sensory neurons. Gene Expr Patterns 10:65-74.

Story GM, Peier AM, Reeve AJ, Eid SR, Mosbacher J, Hricik TR, Earley TJ, Hergarden AC, Andersson DA, Hwang SW, et al. (2003) ANKTM1, a TRP-like channel expressed in nociceptive neurons, is activated by cold temperatures. Cell 112:819-829.

Su D, Zhao H, Hu J, Tang D, Cui J, Zhou M, Yang J, and Wang S (2016) TRPA1 and TRPV1 contribute to iodine antiseptics-associated pain and allergy. EMBO Rep 17: $1422-1430$

Taylor-Clark TE, McAlexander MA, Nassenstein C, Sheardown SA, Wilson S, Thornton J, Carr MJ, and Undem BJ (2008) Relative contributions of TRPA1 and TRPV1 channels in the activation of vagal bronchopulmonary C-fibres by the endogenous autacoid 4-oxononenal. J Physiol 586:3447-3459.

Viswanath V, Story GM, Peier AM, Petrus MJ, Lee VM, Hwang SW, Patapoutian A and Jegla T (2003) Opposite thermosensor in fruitfly and mouse. Nature 423: $822-823$.

Wang S, Shi P, and Wang Y (2013) TRPA1 ion channels in vagal afferent nerves contribute to ventilator-induced lung injury in a rat model. Gen Physiol Biophys 32: 389-394.

Weller K, Reeh PW, and Sauer SK (2011) TRPV1, TRPA1, and CB1 in the isolated vagus nerve-axonal chemosensitivity and control of neuropeptide release. Neuropeptides 45:391-400.

Weng HJ, Patel KN, Jeske NA, Bierbower SM, Zou W, Tiwari V, Zheng Q, Tang Z, Mo GC, Wang Y, et al. (2015) Tmem100 is a regulator of TRPA1-TRPV1 complex and contributes to persistent pain. Neuron 85:833-846.

Wu SW, Fenwick AJ, and Peters JH (2014) Channeling satiation: a primer on the role of TRP channels in the control of glutamate release from vagal afferent neurons. Physiol Behav 136:179-184.

Wu SW, Lindberg JEM, and Peters JH (2016) Genetic and pharmacological evidence for low-abundance TRPV3 expression in primary vagal afferent neurons. Am $J$ Physiol Regul Integr Comp Physiol 310:R794-R805.

Xiao B, Dubin AE, Bursulaya B, Viswanath V, Jegla TJ, and Patapoutian A (2008) Identification of transmembrane domain 5 as a critical molecular determinant of menthol sensitivity in mammalian TRPA1 channels. J Neurosci 28:9640-9651.

Xu H, Delling M, Jun JC, and Clapham DE (2006) Oregano, thyme and clove-derived flavors and skin sensitizers activate specific TRP channels. Nat Neurosci 9: 628-635.

Yu S, Gao G, Peterson BZ, and Ouyang A (2009) TRPA1 in mast cell activationinduced long-lasting mechanical hypersensitivity of vagal afferent C-fibers in guinea pig esophagus. Am J Physiol Gastrointest Liver Physiol 297:G34-G42.

Zhang L, Jones S, Brody K, Costa M, and Brookes SJ (2004) Thermosensitive transient receptor potential channels in vagal afferent neurons of the mouse. Am J Physiol Gastrointest Liver Physiol 286:G983-G991.

Zhao H, Sprunger LK, and Simasko SM (2010) Expression of transient receptor potential channels and two-pore potassium channels in subtypes of vagal afferent neurons in rat. Am J Physiol Gastrointest Liver Physiol 298:G212-G221.

Zhong L, Bellemer A, Yan H, Ken H, Jessica R, Hwang RY, Pitt GS, and Tracey WD (2012) Thermosensory and nonthermosensory isoforms of Drosophila melanogaster TRPA1 reveal heat-sensor domains of a thermoTRP channel. Cell Reports 1:43-55.

Zhou Y, Suzuki Y, Uchida K, and Tominaga M (2013) Identification of a splice variant of mouse TRPA1 that regulates TRPA1 activity. Nat Commun 4:2399.

Address correspondence to: Dr. Shaw-wen "Christine" Wu, Department of Neuroscience, The Scripps Research Institute, 130 Scripps Way C349, Jupiter, FL 33458. E-mail: swu@scripps.edu 